\title{
Investigation of Elastic Energy on Single Crystal GaN Nanobeams with Different Span
}

\author{
Shang-Chao Hung, ${ }^{1}$ Cheng-Fu Yang, ${ }^{2}$ and Yi-Cheng Hsu ${ }^{3}$ \\ ${ }^{1}$ Department of Information Technology \& Communication, Shih Chien University, Kaohsiung Campus, Kaohsiung 845, Taiwan \\ ${ }^{2}$ Department of Chemical and Materials Engineering, National University of Kaohsiung, Kaohsiung 811, Taiwan \\ ${ }^{3}$ Department of Biomechatronics Engineering, National Pingtung University of Science and Technology, Pingtung 912, Taiwan
}

Correspondence should be addressed to Shang-Chao Hung; schung99@gmail.com

Received 15 March 2014; Accepted 15 April 2014; Published 12 May 2014

Academic Editor: Ho Chang

Copyright (C) 2014 Shang-Chao Hung et al. This is an open access article distributed under the Creative Commons Attribution License, which permits unrestricted use, distribution, and reproduction in any medium, provided the original work is properly cited.

\begin{abstract}
This research presents a novel technique which can more efficiently fabricate different spans of nanobeams on the same substrate. It requires less time to prepare specimen and further shortens the process of aligning, clamping, and testing. Also, we probe into the elastic deformation properties of clamped freestanding $\mathrm{GaN}$ nanobeams with different spans. In the bending process, displacement, $D$, corresponding to load, $P$ is strongly dependent on the span of nanobeam at the same penetration depth and a distinct linearity is observed. Young's moduli $E$ of the $\mathrm{GaN}$ in this study are calculated as $171.3 \mathrm{GPa} \pm 5.4 \%$ and $264.2 \mathrm{GPa} \pm 4.7 \%$ by strain energy methods, respectively, for the longer and shorter spans of nanobeams, serving as a simple supporting beam of elastic material under small deformation. The result shows that, even under small deformation, the rigidity enhancement helps the shorter nanobeam store more elastic energy.
\end{abstract}

\section{Introduction}

Since the conversion of mechanical energy into electric one has been realized as a nanogenerator for the recycle energy issue, Nitride-based one-dimensional nanomaterials have attracted much attention $[1,2]$. Among them, the materials AlN, InN, and GaN are usually fabricated by bottom-up growing techniques and the top-down growing methods also involve in some GaN-based nanogenerator fabrication [3]. This means that nanogenerator can be realized by semiconductor processing techniques. In nanogenerator experiment in which the kinetic energy is transferred into the elastic one, it is interesting to note that the output current is significantly dependent on the bended nanorods with different aspect ratio whereas the magnitudes of the deflecting kinetic energy are equal [3]. Furthermore, the deflecting direction is perpendicular to the axial direction (c-plane of $\mathrm{GaN}$ film) of nanorods which were stretched on the outer surface and were compressed on the inner surface [4], which consequently creates the rigidity. The shorter aspect ratio will produce more rigidity than that in longer one at the same displacement. This means that the shorter a nanorod is, the more energy is needed to bend a nanorod. Therefore, considering its elastic behavior, the shorter nanorod can store more energy. The mechanical characterization of onedimensional nanostructure with different aspect ratio has proven itself a fertile ground for further research. However, their sizes and configurations, which cannot be precisely tested by conventional measures, have posed great challenges to the mechanical characterization of nanostructures. To acquire better measurement, axial loading for uniaxial compression is adopted in experiments concerning mechanical behavior about one-dimensional $\mathrm{GaN}$ nanomaterials $[5,6]$. In this method, the advanced group III nitride semiconductor nanotube fabrication technology makes intricate manipulations unnecessary. Besides, precise alignment of an individual nanotube is still a challenging task. Various novel methods have been developed to overcome the aligning problem, such as microelectromechanical system (MEMS) test [7-9], micro/nanoindentation tests [10-13], tensile tests [14], and bending tests [15-17], each of which has its contribution and advantages. Unfortunately, aligning, clamping, and testing of 
individual nanowire between two fixed ends still demand great specimen preparation time.

To offer a better solution, this report presents a novel, more efficient technique which can fabricate different spans of nanobeams on a homogeneous substrate. It requires less time to prepare specimen and shortens the process of aligning, clamping, and testing of individual nanobeam between two fixed ends. In this study, nanobeams, fabricated by focused ion beam (FIB, FEI Nova-200 NanoLab Compatible) with different spans, are clamped in between two homogeneous fixed ends as simple supporting beams. They are subjected to tension or compression loads in an in situ transmission electron microscopy. These bending tests of nanobeams install a conical tip inside the transmission electron microscopy (TEM) chamber, improving the visibility for alignment, load, and displacement. In addition to testing the elastic energy of nanobeams, we also conduct experiments for the linear relationship of stress and strain with different spans.

\section{Experiment}

Firstly, a slice of $\mathrm{GaN}$ with a feature size $\sim 250 \mathrm{~nm}$ in width, $10 \mu \mathrm{m}$ in length, and $3 \mu \mathrm{m}$ in depth was peeled from single crystal GaN film by FIB (FEI Nova-200 NanoLab Compatible) at $5 \mathrm{keV}$. The single crystal GaN film was grown as prior study showed in a standard process by metal organic chemical

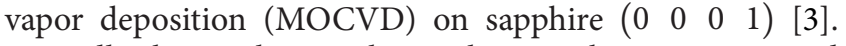
Secondly, this single crystal GaN slice was then postpatterned and machined by FIB again with a tilted angle of $30^{\circ}$ to generate a uniform set of suspended bridge structures of different lengths.

After fabrication, the geometric parameters of each nanobeam on the GaN substrate were measured from scanning electron microscope (SEM) observations. The length ranges are $\sim 1.5$ and $\sim 2.0 \mu \mathrm{m}$, while the width is $z=\sim 250 \mu \mathrm{m}$. The thickness of the GaN nanobeams was examined by TEM cross-section observation to be $y=\sim 50-195 \mathrm{~nm}$ while the as-deposited thickness ( $y$-direction) was average $125 \mathrm{~nm}$ and formed a hexagonal columnar grain structure in $c$-axis.

The fabrication process and cross-sectional structures of GaN nanobeams are depicted in Figure 1. The right part of Figure 1 reveals the transverse geometry of the fabricated nanobeam. The trapezoid shape is formed by the FIB machining of the GaN substrate. Surface morphologies and crosssectional views of these two GaN nanobeam samples were then examined by SEM and TEM.

Figures 2(a) and 2(b) are the tilted top view SEM images, showing the fabricated nanobeam samples with different length. The nanobeam length, $L$, is defined as the suspending distance between two tops of supporting leg with column-like ends. Therefore, the $2 \mu \mathrm{m}$ and $1.5 \mu \mathrm{m}$ lengths of nanobeams are with aspect ratios of $\sim 10$ and $\sim 7$, respectively. In situ TEM was then performed to observe and evaluate the mechanical behavior of the GaN nanobeams with different aspect ratio. After the vertical alignment was completed, the displacement between the specimen and conical tip was measured by using a TEM with the loading device installed, given that TEM can precisely locate interfaces and sharply measure

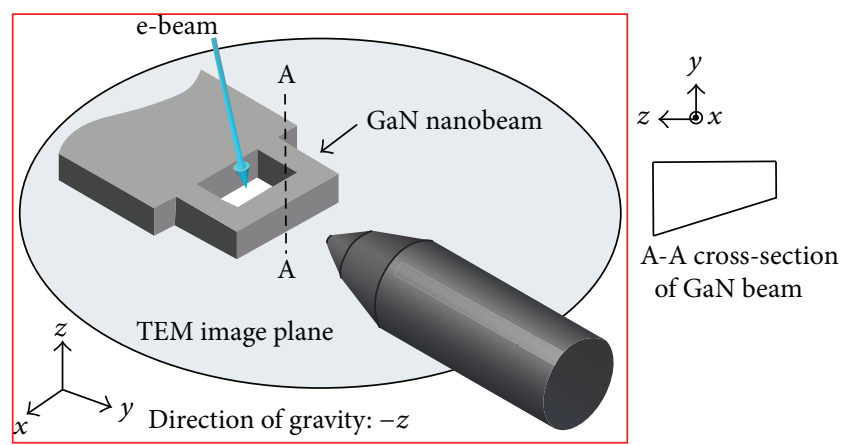

FIgURE 1: A schematic illustration showing a single crystal GaN nanobeam machined by FIB and a nanomechanical test and loading direction of a nanobeam in TEM. The right part is a cross-section view of GaN nanobeam with a cross-section area of $0.03125 \mu \mathrm{m}^{2}$ after machining by FIB.

the displacement and contact point of the loading tip. All observations are conducted in an accelerating voltage of $15 \mathrm{kV}$ under vacuum $\left(1.5 \times 10^{-5} \mathrm{~Pa}\right)$. When the in situ experiment is being exercised, the loading rate of $10 \mathrm{nms}^{-1}$ is applied continuously to load the tested specimen and then unloaded at the same rate while a preset maximum load displacement of $200 \mathrm{~nm}$ was reached.

\section{Result and Discussion}

Figures 3(a) and 3(b) show the loading-unloading characteristics of the nanoindentation test, targeting these two different spans of single crystal GaN nanobeams with an aspect ratio of $\sim 10$ (i.e., sample I) and an aspect ratio of $\sim 7$ (i.e., sample II), operating in in situ TEM at room temperature, respectively, whereas the (a)-(b)-(c)/(c)-(d)-(e) symbols denote the experimental loading/unloading data, respectively.

It is found that the maximum loading forces on sample I and sample II in penetration indentation depth of $\sim 200 \mathrm{~nm}$ (point (c) of the loading curve) were $\sim 30 \mu \mathrm{N}$ and $\sim 172 \mu \mathrm{N}$, respectively. However, the eventful slope changes transpire in point (b) of Figures 3(a) and 3(b) when the loads get beyond $\sim 8 \mu \mathrm{N}$ and $\sim 20 \mu \mathrm{N}$, while the maximum linear deflections are $90 \mathrm{~nm}$ and $80 \mathrm{~nm}$ in sample I and sample II, respectively. The linear elastic behavior of both nanobeam specimens became nonlinear when the loading force was continuingly increased beyond the point (b) in Figures 3(a) and 3(b). The sudden increase of slope suggests the existence of more complex elastic and plastic behaviors, particularly on the shorter span of GaN nanobeam system (sample II). In the shorter one (sample II), the slope was found to increase dramatically from $\sim 20 \mu \mathrm{N}$ to $\sim 172 \mu \mathrm{N}$ while the penetration depth is increasing from $80 \mathrm{~nm}$ to $200 \mathrm{~nm}$ as shown on point (b) to point (c) in Figure 3(b). Clearly, shorter specimen, namely, sample II, needs more load and work than longer ones to be pressed to the same depth of the pressed sample I. In indentation test, longer GaN nanobeam specimen shows more linear elasticity than shorter ones as shown in the contrast of sample I and sample II. 


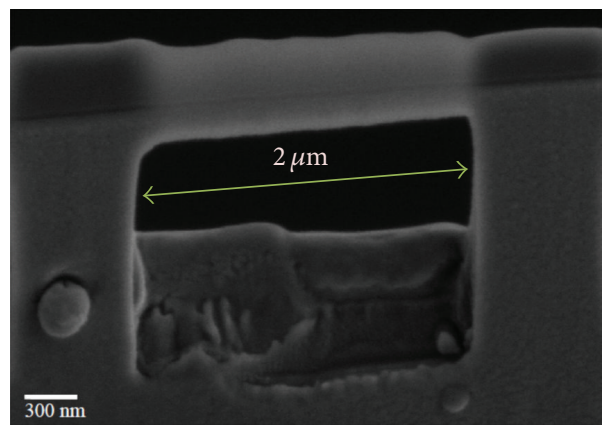

(a)

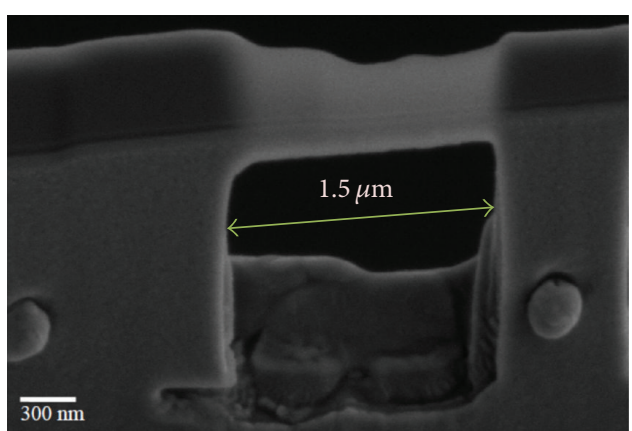

(b)

FIGURE 2: Tilted $45^{\circ}$ top-view FESEM images of nanobeams with $2.0 \mu \mathrm{m}$ (a) and $1.5 \mu \mathrm{m}$ (b) in length and $250 \mathrm{~nm}$ in width after being machined by FIB.

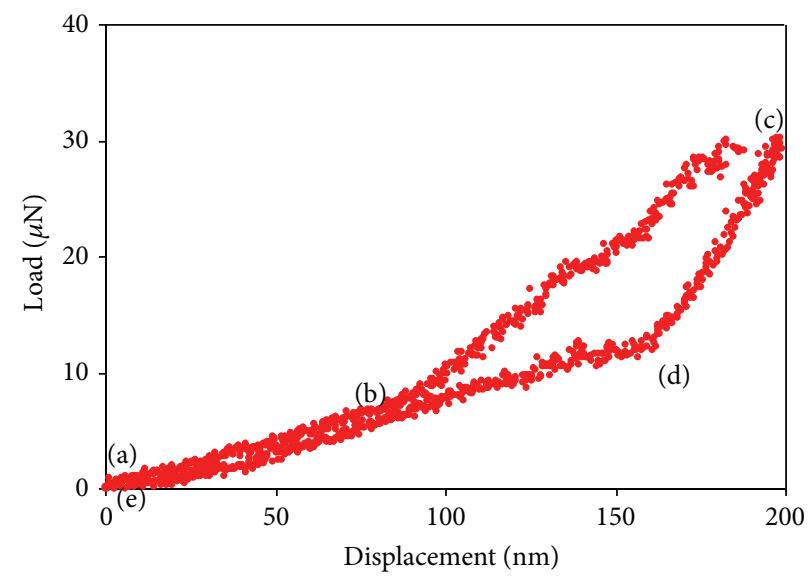

(a)

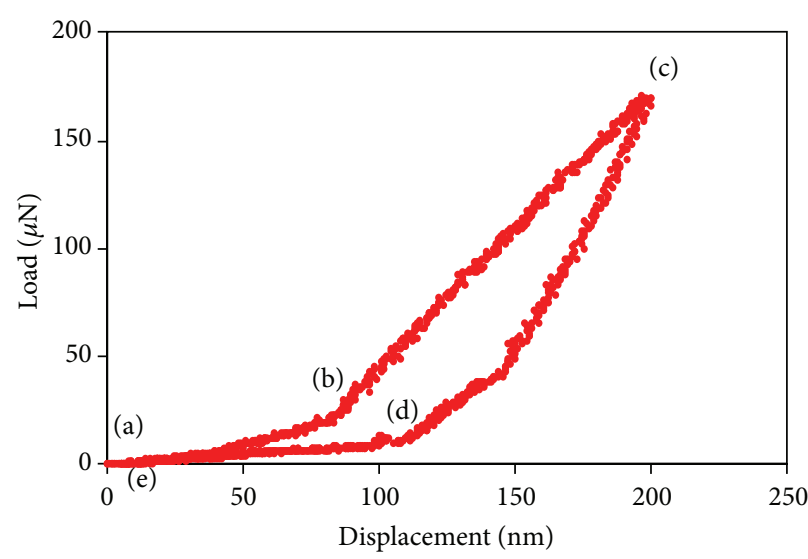

(b)

FIGURE 3: Experimentally observed loading and unloading behavior by indenting on the center of the span of $2.0 \mu \mathrm{m}$ (a) and $1.5 \mu \mathrm{m}$ (b) of $\mathrm{GaN}$ nanobeams with equal indentation depth using a conical indenter in TEM.

More interestingly, both unloading curves return approximately to the original position. The perfect retreats of the loading and unloading curves indicate that the elastic energy can be stored and released in both of the indentation tests of the freestanding nanobeam systems without considering the aspect ratio.

As we know, axial tensile force is also introduced when the nanobeam systems are displaced at the center section in this study. Therefore, the enhancement of nanobeam rigidity and nonlinearity in force versus displacement variation is transpired [18]. Furthermore, assuming the prestretch was produced when the film was being grown by MOCVD, in a nanobeam fabrication can be neglected in the same specimen, the load-deflection behaviors in a given indentation test will be affected by four factors [19]: geometrical nonlinearity introduced by kinematic effects, plastic deformation, increasing contact area during indentation, and rate dependent material effect. Fortunately, limiting displacements in the bending test can minimize the influence of these four factors.

Given the elastic behavior shown in the experimental data, when at low displacement, the loading force of both nanobeams increased linearly with the displacement. This means that both $\mathrm{GaN}$ nanobeam systems exhibited linear elastic characteristics, when they were operated in low displacement. Under large deformation, the theoretical bases of the nanobeam test obey the classical beam theory with concern in substrate deformation [20].

Under small deformation (line (a)-(b) in Figures 3(a) and $3(\mathrm{~b})$ ), the deflection, $D$, is linearly proportional to the lateral load, $P$. Therefore, we define the slope, $k$. On the other hand, for small deformation of unloading curves (line (d)(e) in Figures 3(a) and 3(b)), the data of the recovery also exhibit a linear relationship between unload and deflection. We define the slope, $j$. It is found that the slope $j$ is almost the same as the slope $k$ in Figure 3(a), further suggesting a good linear elastic behavior observed in higher aspect ratio of GaN nanobeam system, compared to that in lower aspect ratio of $\mathrm{GaN}$ nanobeam system.

For a given nanobeam sample with available values of sample length and thickness, the linear relationship between stress and strain in simple tension or compression can be expressed by the equation $P=k D$, in which $D, P$, and $k$ are displacement, correspondence to load, and a constant of proportionality, respectively. The equation is commonly 
TABLE 1: The experimentally measured data and calculating results of GaN nanobeam systems with different span.

\begin{tabular}{lccccc}
\hline Systems & Span & Deflection displacement & Loading force & Moment of inertia $I$ on $z$-axis & Young's modulus $E$ \\
\hline Sample I & $2 \mathrm{um}$ & $90 \mathrm{~nm}$ & $8.01 \pm 0.23 \mu \mathrm{N}$ & $8.64 \times 10^{-5} \mu \mathrm{m}^{4}$ & $171.3 \mathrm{GPa} \pm 5.4 \%$ \\
Sample II & $1.5 \mathrm{um}$ & $80 \mathrm{~nm}$ & $20.32 \pm 0.43 \mu \mathrm{N}$ & $6.65 \times 10^{-5} \mu \mathrm{m}^{4}$ & $264.2 \mathrm{GPa} \pm 4.7 \%$ \\
\hline
\end{tabular}

known as Hook's law, a rule only applied to ordinary tension and compression. Young's modulus is then obtained by measuring the nanobeam displacement as a function of the applied load.

For a two-end fixed beam used here, Young's modulus cannot be determined from merely a slope of deflection versus load. We have to also consider linear elastic beam bending theory. The possibility of linear elastic deformation is discussed below. We assume that the GaN nanobeam systems are simple supporting beam systems, subjected to a vertical loading $P$ ( $y$-direction). The linear force $P$ is proportional to $y$ and its value is $P_{0}$ when $y=y_{0}$. Then $P=\left(P_{0} / y_{0}\right) y d y$, and the work is

$$
W=\int_{0}^{y_{0}}\left(\frac{P_{0}}{y_{0}}\right) y d y \frac{P_{0} y_{0}}{2} .
$$

When an elastic material is deformed, this work can be stored in the form of potential energy. In this case, potential energy is strain energy $U$ which can be expressed by the equation

$$
U=\frac{P^{2} L^{3}}{96 E I}=W=\frac{P_{0} y_{0}}{2} .
$$

The displacement is

$$
y_{0}=\frac{P L^{3}}{48 E I},
$$

where $I, E$, and $L$ are the moment of inertia, Young's modulus, and span of the clamped portion of the beam, respectively. The moments of inertia on $z$-axis of nanobeam systems are calculated as $I=8.64 \times 10^{-5} \mu \mathrm{m}^{4}$ and $6.65 \times 10^{-5} \mu \mathrm{m}^{4}$ for the lengths of $2.0 \mu \mathrm{m}$ (sample I) and $1.5 \mu \mathrm{m}$ (sample II), respectively. The beam's deflections at the point where $P$ is applied are $90 \mathrm{~nm}$ and $80 \mathrm{~nm}$.

The force is assumed to change linearly as a function of bending displacement when the experiment was conducted on the curve of point (a) to point (b) in Figures 3(a) and $3(\mathrm{~b})$. The average loading forces on deflection displacement of $90 \mathrm{~nm}$ and $80 \mathrm{~nm}$ are obtained as $8.01 \pm 0.23 \mu \mathrm{N}$ and $20.32 \pm 0.43 \mu \mathrm{N}$, respectively. Therefore, we can estimate Young's modulus $E$ from formula (3) by equating the work done to the strain energy in the nanobeam. Young's modulus $E$ in this study is calculated as $171.3 \mathrm{GPa} \pm 5.4 \%$ and $264.2 \mathrm{GPa}$ $\pm 4.8 \%$ for the longer nanobeam system (sample I) and shorter nanobeam system (sample II), respectively. Table 1 depicts the data and calculating results of $\mathrm{GaN}$ nanobeam systems with different spans. It is found that the enhancement of nanobeam rigidity, a byproduct of axial tensile force, is strongly dependent on the span of nanobeam even under small deformation. Notably, the axial direction of a deflected nanobeam in this experiment is not on $c$-axis of $\mathrm{GaN}$ film examined in nanogenerator studies but is perpendicular to the $c$-axis of nanobeam.

A series of in situ TEM images of sample I, as shown in Figure 4, presents the elastic-plastic behavior described by the data of Figure 3(a). Figure 4(a) shows the nanobeam before the deflecting experiment. When the tip gradually loads downward to the nanobeam at the beginning of the nanoindentation, the conical tip can easily bend the clamped $\mathrm{GaN}$ nanobeam because it is freestanding, with no barrier to restrict the bending process.

When a certain bending indentation depth has been achieved, as shown on point (b) in Figure 3(a) corresponding to Figure 4(b), a dramatic slope change occurs. Such change later brings about rigidity when the loading exceeds the linear elastic of the nanobeam. At this moment, the property of the nanomechanical action starts to change: the linear beam-like behavior is now turned into a nonlinear one.

The maximum loading force deforming the nanobeam by the conical tip on point (c) in Figure 3(a) is given in Figure 4(c). Here, the relative location between conical tip and nanobeam can be easily targeted. This image shows that the loading force is not a perfectly concentrated load, suggesting the transition from single point bending indentation of a beam to surface stretching indentation. Moreover, the locations which are not contacted by the conical tip possess good elastic recovery.

Another dramatic slope switch can be found in Figure 4(d) corresponding to point (d) in Figure 3(a). Figure 4(e) corresponding to point (e) in Figure 3(a) shows the finished view of the deflected nanobeam, as expected, in which the nanobeam recovered perfectly to the original location as shown in Figure 4(a).

\section{Conclusions}

This research investigates a novel technique which can fabricate different span of nanobeams on a substrate and it does not require significant amounts of specimen preparation time for aligning, clamping, and testing of individual nanobeam between two fixed ends. In this study, nanobeams fabricated by focused ion beam with different spans are clamped in between two homogeneous fixed ends as simple supporting beams, subjected to load in an in situ TEM. These in situ TEM analyses provide details of bending to stretching-induced mechanical deformation.

It is found that a more conspicuous linear elastic behavior is observed in indentation test of longer $\mathrm{GaN}$ nanobeam specimen (sample I). Young's moduli have also been studied by strain energy method with presuming GaN nanobeams as simply supported beam systems of elastic material. The slopes of the $L-D$ curves standing for the linear relationship between 


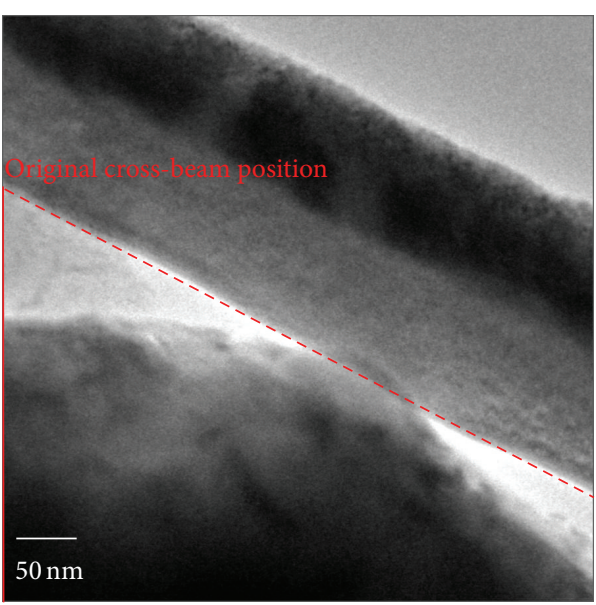

(a)

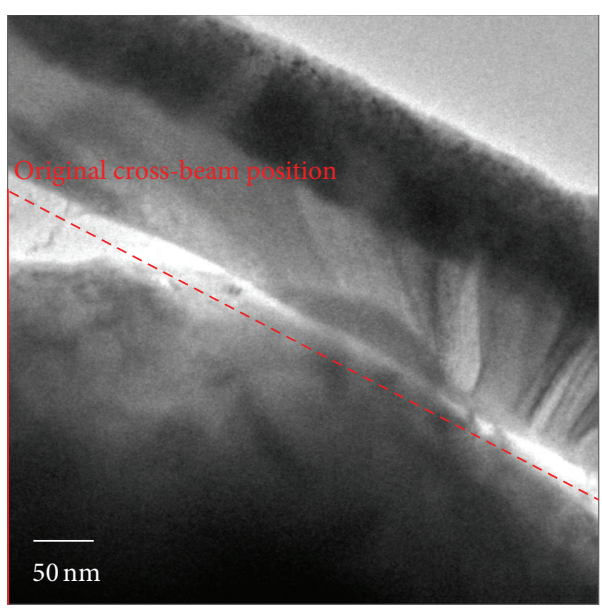

(c)

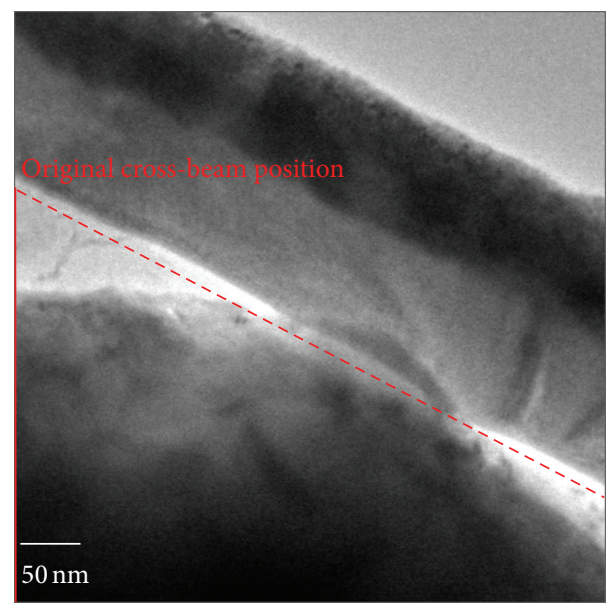

(b)

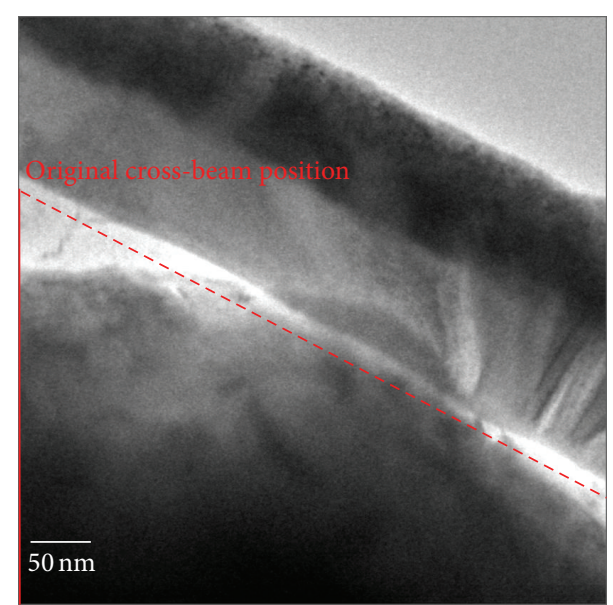

(d)

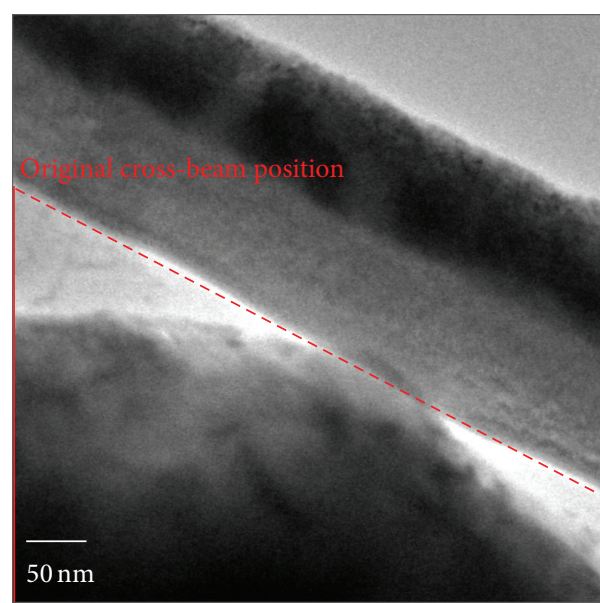

(e)

FIgURE 4: A sequence of images extracted from a video recording of sample I, corresponding to the indentation experiment performed in point (a) to point (e) of Figure 3(a), showing the deformation stages: (a) the GaN nanobeam before loading, (b) at the end of linear loading, (c) the bended nanobeam at peak load, (d) during unloading at the end of nonlinear or plastic deformation, and (e) nearly no residual deformation after full separation. 
stress and strain at the initial phase of tests prove that the enhancement of nanobeam rigidity is strongly dependent on the span of nanobeam even under small deformation.

\section{Conflict of Interests}

The authors declare that there is no conflict of interests regarding the publication of this paper.

\section{Acknowledgments}

This work was supported by the National Science Council of Taiwan under Contract nos. NSC 99-2221-E-158-009, NSC 100-2221-E-158-002, and NSC 102-2221-E-020-016 and the Shih Chien University Kaohsiung Campus (USC-102-0505017 and USC-102-08-01006). The authors' thanks also go to the Chemical and Materials Engineering Department of National University of Kaohsiung, Center for Micro/Nano Science and Technology of National Sun Yat-Sen University for the equipment support, and Dr. Shang Chao Hung for technical support.

\section{References}

[1] W. S. Su, Y. F. Chen, C. L. Hsiaoand, and L. W. Tu, "Generation of electricity in GaN nanorods induced by piezoelectric effect," Applied Physics Letters, vol. 90, no. 6, Article ID 063110, 2007.

[2] X. Wang, J. Song, F. Zhang, C. He, Z. Hu, and Z. Wang, "Electricity generation based on one-dimensional group-III nitride nanomaterials," Advanced Materials, vol. 22, pp. 21552160, 2010.

[3] S.-C. Hung, "Generation of piezoelectricity by deflecting nanorods vertically on GaN template," Journal of the Electrochemical Society, vol. 158, no. 12, pp. H1265-H1269, 2011.

[4] Z. L. Wang and J. Song, "Piezoelectric nanogenerators based on zinc oxide nanowire arrays," Science, vol. 312, no. 5771, pp. 243246, 2006.

[5] S.-C. Hung, Y.-K. Su, T.-H. Fang, and S.-J. Chang, "Shell buckling behavior investigation of individual gallium nitride hollow nanocolumn," Applied Physics A, vol. 84, no. 4, pp. 439443, 2006.

[6] S.-C. Hung, Y.-K. Su, T.-H. Fang, S.-J. Chang, and L.-W. Ji, "Buckling instabilities in GaN nanotubes under uniaxial compression," Nanotechnology, vol. 16, no. 10, pp. 2203-2208, 2005.

[7] M. Naraghi, I. Chasiotis, H. Kahn, Y. Wen, and Y. Dzenis, "Novel method for mechanical characterization of polymeric nanofibers," Review of Scientific Instruments, vol. 78, no. 8, Article ID 085108, 2007.

[8] M. Naraghi, T. Ozkan, I. Chasiotis, S. S. Hazra, and M. P. de Boer, "MEMS platform for on-chip nanomechanical experiments with strong and highly ductile nanofibers," Journal of Micromechanics and Microengineering, vol. 20, no. 12, Article ID 125022, 2010.

[9] Y. Zhu and H. D. Espinosa, "An electromechanical material testing system for in situ electron microscopy and applications," Proceedings of the National Academy of Sciences of the United States of America, vol. 102, no. 41, pp. 14503-14508, 2005.

[10] S. M. Han, R. Saha, and W. D. Nix, "Determining hardness of thin films in elastically mismatched film-on-substrate systems using nanoindentation," Acta Materialia, vol. 54, no. 6, pp. 15711581, 2006.

[11] G. M. Pharr and W. C. Oliver, "Measurement of thin film mechanical properties using nanoindentation," MRS Bulletin, vol. 17, pp. 28-33, 1992.

[12] F. Yang and J. C. M. Li, "Adhesion of a rigid punch to an incompressible elastic film," Langmuir, vol. 17, no. 21, pp. 65246529, 2001.

[13] T.-Y. Zhang, L.-Q. Chen, and R. Fu, "Measurements of residual stresses in thin films deposited on silicon wafers by indentation fracture," Acta Materialia, vol. 47, no. 14, pp. 3869-3878, 1999.

[14] H. D. Espinosa, B. C. Prorok, and M. Fischer, "A methodology for determining mechanical properties of freestanding thin films and MEMS materials," Journal of the Mechanics and Physics of Solids, vol. 51, no. 1, pp. 47-67, 2003.

[15] J. Schweitz, "Mechanical characterization of thin films by micromechanical techniques," MRS Bulletin, vol. 17, pp. 34-45, 1992.

[16] M. J. Kobrinsky, E. R. Deutsch, and S. D. Senturia, "Effect of support compliance and residual stress on the shape of doubly supported surface-micromachined beams," Journal of Microelectromechanical Systems, vol. 9, no. 3, pp. 361-369, 2000.

[17] T.-Y. Zhang, Y.-J. Su, C.-F. Qian, M.-H. Zhao, and L.-Q. Chen, "Microbridge testing of silicon nitride thin films deposited on silicon wafers," Acta Materialia, vol. 48, no. 11, pp. 2843-2857, 2000.

[18] A. Heidelberg, L. T. Ngo, B. Wu et al., "A generalized description of the elastic properties of nanowires," Nano Letters, vol. 6, no. 6, pp. 1101-1106, 2006.

[19] K. C. Maner, M. R. Begley, and W. C. Oliver, "Nanomechanical testing of circular freestanding polymer films with sub-micron thickness," Acta Materialia, vol. 52, no. 19, pp. 5451-5460, 2004.

[20] S. P. Timoshenko and S. Woinowsky-Krieger, Theory of Plates and Shells, McGraw-Hill, New York, NY, USA, 1959. 

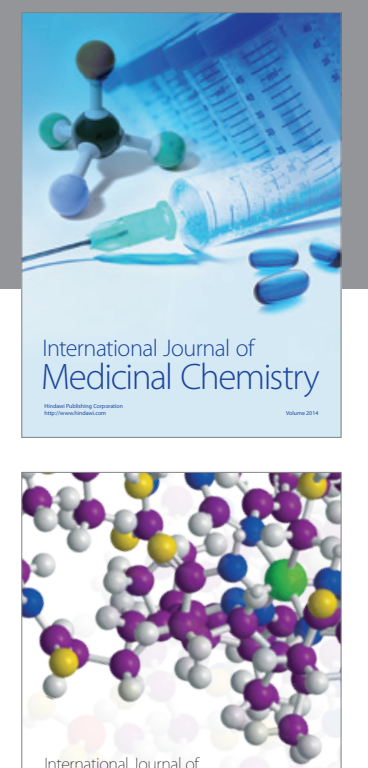

\section{Carbohydrate} Chemistry

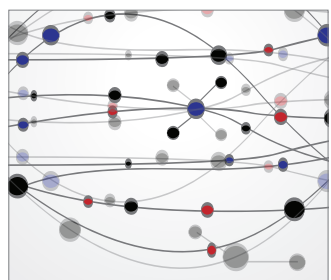

The Scientific World Journal
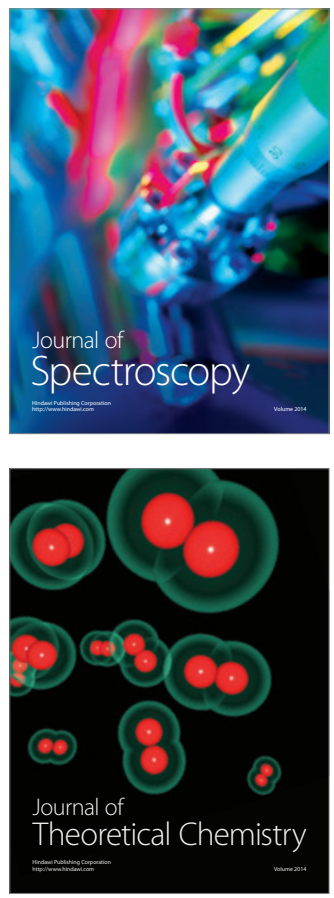
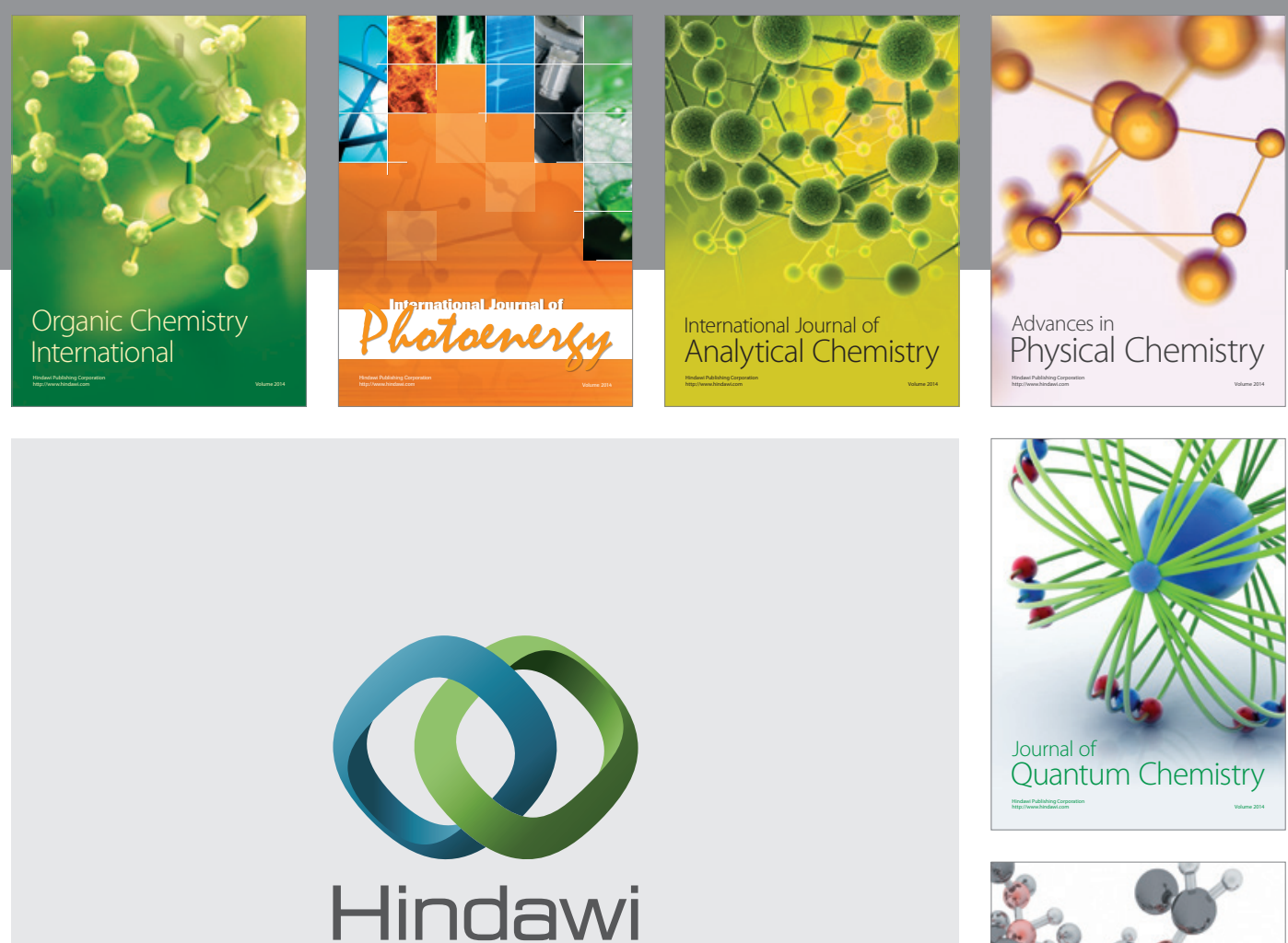

Submit your manuscripts at

http://www.hindawi.com

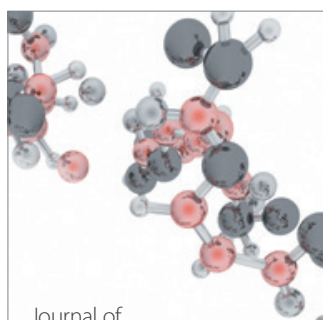

Analytical Methods

in Chemistry

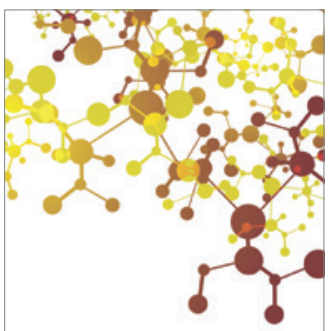

Journal of

Applied Chemistry

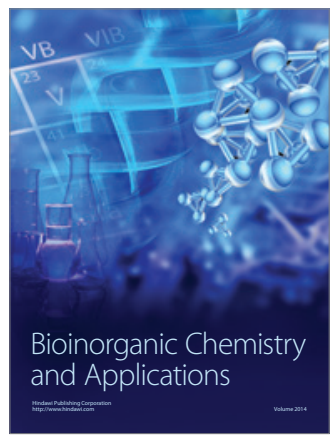

Inorganic Chemistry
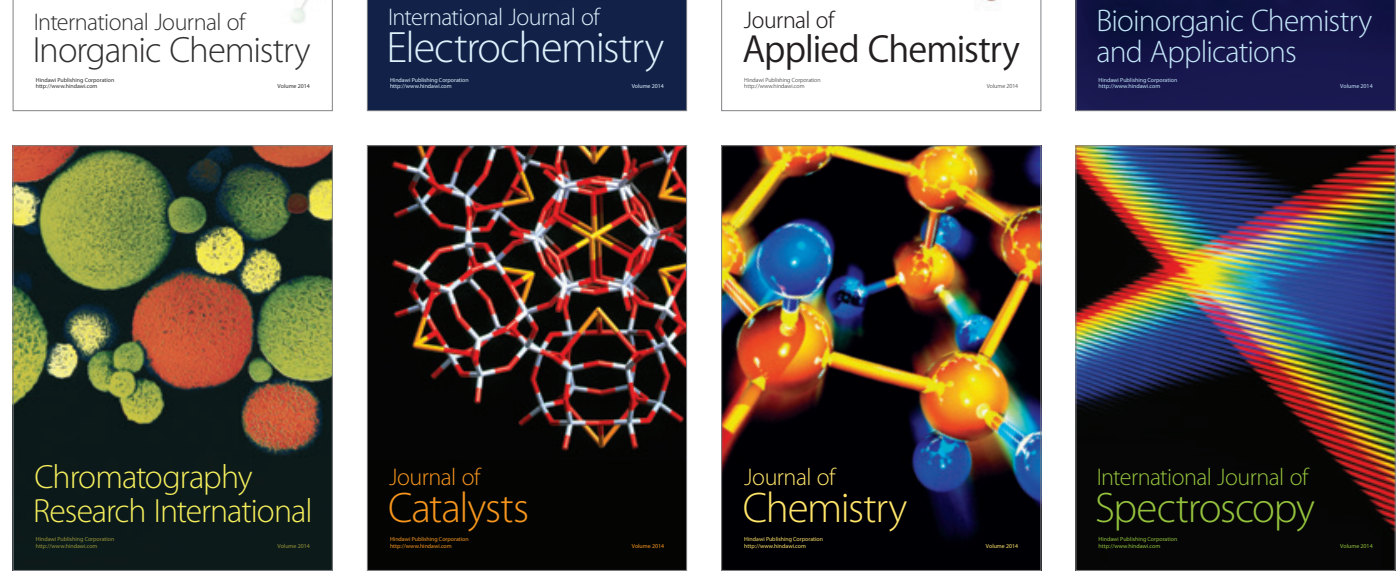